\title{
Sound arguments, true premises, and valid conclusions
}

\author{
Thoralf M. Sundt, MD
}

See related article on pages 2060-9.

The human understanding, once it has adopted an opinion, collects any instances that confirm it, and though the contrary instances may be more numerous and more weighty, it either does not notice them or else rejects them.

Francis Bacon, Novum Organum

The group from Northwestern University reports outstanding outcomes for the management of aortic dilatation associated with BAV as directed by the 2010 American College of Cardiology/American Heart Association guidelines for the diagnosis and management of patients with thoracic aortic disease. With a mortality rate less than $1 \%$, the results speak for themselves. Their series join a number of others demonstrating that in experienced hands, aortic root replacement can be accomplished at low risk. The importance of experience clearly has been demonstrated using the Society of Thoracic Surgeons database, with operative mortality rate in low-volume centers almost double that in high-volume centers. It is important to add that even in large-volume centers, the operative mortality rate was $3.4 \%$. The authors of the current study argue that their data "validate current guidelines."

The issue being addressed here-the validity of the stated threshold for intervention on BAV aortopathy-is of enormous clinical and social importance. Given the incidence of BAV and associated aortic enlargement, it is quantitatively significant. Given the young age of those affected, whether a young woman contemplating pregnancy or the family "breadwinner" whose occupation demands heavy lifting or whose life insurance is at risk of being denied, it is also emotionally impactful. There is very real economic impact as well, with the proportion of concomitant aortic procedures and associated costs more than doubling over the last decade. The answer to this question matters personally to surgeons too, as we all tend to feel a burden of responsibility for the long-term outcome of patients once

From the Division of Cardiac Surgery, Massachusetts General Hospital, Harvard Medical School, Boston, Mass.

Disclosures: Author has nothing to disclose with regard to commercial support.

Received for publication Sept 18, 2014; accepted for publication Sept 19, 2014

Address for reprints: Thoralf M. Sundt, MD, Division of Cardiac Surgery, Massachusetts General Hospital, Harvard Medical School, 55 Fruit St, Boston, MA 02114

(E-mail: tsundt@mgh.harvard.edu).

J Thorac Cardiovasc Surg 2014;148:2070-1

$0022-5223 / \$ 36.00$

Copyright (c) 2014 by The American Association for Thoracic Surgery

http://dx.doi.org/10.1016/j.jtcvs.2014.09.056 we have met them. True, we did not give them the condition, but somehow we feel responsible nonetheless.

The crucial question here is whether these data "validate" the guidelines?

In a formal sense, a sound argument is based on premises that are true and conclusions that are valid. In this case, the premise demonstrated by these data is that "aortic replacement can be accomplished at low risk." It is also certainly true that aortic dissection is prevented by aortic replacement. Accordingly, one may validly conclude that aortic dissection can be prevented at low risk. But we need more than formally valid arguments; we need clinically meaningful conclusions. To arrive at the conclusion that the guidelines are valid, one really must demonstrate that more lives will be saved than lost by adherence. As such, the second premise in which we are most interested must be that "the risk of aortic dissection without surgery is high," at least higher than the risk of the surgery. This study does not address this question. True, a number of other studies are referenced that argue this point, but of late, an increasing number of studies argue the opposite. We cannot directly measure the dissections that we prevent, but we can get an idea of the population risk of dissection among similar patients. At this point, I think that the truth is we do not know (level of evidence "C"). We just need to recognize our ignorance.

Guidelines are increasingly popular. They are certainly useful as we struggle with drawing the line-balancing the risk and rewards-on aortic intervention. As surgeons, we are asked to make binary decisions on the basis of continuous risk variables all the time. We understand that there is no "bright line" short of which the patient is safe and beyond which the patient is suddenly at high risk for dissection; still, we must make that recommendation at some discrete point in time when we believe our patient has crossed that imaginary line. The decisions may seem less dramatic when deciding whether to add an aortic intervention to a planned aortic valve replacement than when the principle indication for operation is the aorta itself. But these 2 questions have become, in practice, intertwined with conjecture and inference made in one setting on the basis of the other. That is understandable; it is how we are built as human decision-makers. We frequently think by analogy.

Still, we need to recognize that there is also a line that we may cross separating benefit from harm. We may not be sure where that line lies, but somewhere; if we are too aggressive, we will surely hurt more people than we help. Aortic intervention is not risk-free (note that even in this series, 
reoperation for bleeding was twice as common among those with intervention for an aorta 45-49 $\mathrm{mm}$ than for aortic valve replacement alone). We need to know more than operative risk; we need to know something about the benefits derived.
In essence, this study addresses safety but not efficacy. Despite the prevailing enthusiasm for a more aggressive approach to the BAV, we should resist the urge to overextending these findings to confirm what we already think we know. 